\title{
ORIGINAL ARTICLE PKR negatively regulates leukemia progression in association with PP2A activation, Bcl-2 inhibition and increased apoptosis
}

\author{
X Cheng ${ }^{1}$, RL Bennett ${ }^{1}$, X Liu, M Byrne and W Stratford May
}

Reduced expression and activity of the proapoptotic, double-stranded RNA-dependent protein kinase, PKR (protein kinase R) is observed in breast, lung and various leukemias, suggesting that loss of PKR potentiates transformation. Now we report that decreased PKR activity inhibits chemotherapy-induced apoptosis of leukemia cells both in vitro and in vivo. Inhibition of PKR expression or activity reduces protein phosphatase 2A (PP2A) activity, a B-cell lymphoma 2 (Bcl-2) phosphatase, resulting in enhanced $\mathrm{Bcl}-2$ phosphorylation. Thus, inhibition of PKR activity leads to hyperphosphorylation of $\mathrm{Bcl}-2$, stabilization of Bcl-2/Bax interaction and decreased Bax insertion into the outer mitochondrial membrane. Treatment with the PP2A activator, FTY720, restores $\mathrm{BCl}-2$ dephosphorylation and apoptosis in cells with reduced PKR expression following stress. Significantly, xenografts of REH leukemic cells with reduced PKR display significantly increased tumor volume, increased resistance to doxorubicin treatment and shorter survival. Importantly, FTY720 treatment restores sensitivity to chemotherapy and prolongs overall survival of these mice. Collectively, these findings suggest that PP2A activation is a downstream target of PKR and the PKR/PP2A signaling axis is required for rapid and potent stress-induced apoptosis. Importantly, loss of PKR promotes leukemia progression and may serve as a biomarker for predicting chemosensitivity.

Blood Cancer Journal (2013) 3, e144; doi:10.1038/bcj.2013.42; published online 6 September 2013

Keywords: leukemia; PKR; apoptosis; Bcl-2; PP2A

\section{INTRODUCTION}

In hematopoietic stem/progenitor cells, the interferon-inducible, double-stranded RNA-dependent protein kinase, PKR (protein kinase R), is a central mediator for the antiproliferative effects of a wide range of hematopoietic cellular stresses including viral infection, hematopoietic growth factor deprivation, inflammatory cytokines, Toll-like receptor ligands and chemotherapy treatment. $^{1-6}$ Once activated by cellular stress, PKR inhibits proliferation and initiates apoptosis by phosphorylation of eukaryotic initiation factor $2 \alpha$ (elF $2 \alpha$ ) to inhibit new protein synthesis, inhibition of B-cell lymphoma $2(\mathrm{Bcl}-2)$ function and activation of signaling pathways including p38 mitogen-activated protein kinase, nuclear factor- $\kappa \mathrm{B}, \mathrm{p} 53$ and signal transducer and activator of transcription 1.,3,7-10 Loss of PKR expression/activity has been associated with more aggressive human breast carcinoma, non-small-cell lung cancer and various acute and chronic leukemias including B-cell chronic lymphocytic leukemia and T-cell acute lymphoblastic leukemia.,5,11-18 In addition, although PKR expression has been reported to be increased in some solid tumors such as breast, PKR activity is inhibited either directly or indirectly in these cells, resulting in prosurvival conditions. ${ }^{17-19}$ Taken together, these findings suggest that loss of PKR is an important driver of tumorigenesis. ${ }^{14,16,20-22}$

Deregulation of apoptosis is a major contributor to the development of malignant phenotype and chemoresistance in hematopoietic cells. ${ }^{23}$ Importantly, it has been reported that loss of PKR activity may lead to deregulation of apoptosis that promotes and/or maintains the malignant phenotype, but the mechanism remains unclear. Recently, protein phosphatase $2 \mathrm{~A}$
(PP2A) was reported to be a substrate of PKR, and PP2A activation has been reported to regulate the intrinsic apoptosis pathway by directly dephosphorylating $\mathrm{BCl}-2 .{ }^{8,24}$ As studies indicate that PP2A-mediated dephosphorylation of $\mathrm{Bcl}-2$ is critical to initiate apoptosis, and $\mathrm{Bcl}-2$ is commonly overexpressed in hematologic malignancies, we focused our studies on elucidating the role for PKR in regulating apoptosis in human leukemia cells. ${ }^{25-29}$ Significantly, our results indicate that expression of enzymatically active PKR is required for PP2A activation and apoptosis in response to cellular stress including hydrogen peroxide $\left(\mathrm{H}_{2} \mathrm{O}_{2}\right)$ or doxorubicin (DOX) treatment. Furthermore, cells with reduced PKR activity display increased phosphorylated $\mathrm{BCl}-2$, increased association of $\mathrm{Bcl}-2$ with $\mathrm{Bax}$, reduced $\mathrm{Bax}$ insertion into the outer mitochondria membrane (OMM) and enhanced survival following stress treatment. A xenograft model of human leukemia demonstrates that acute leukemia cells with reduced PKR proliferate more rapidly and are resistant to chemotherapy in vivo. These results establish that the PKR/PP2A signaling axis negatively regulates leukemia progression and is required for sensitivity of leukemia cells to chemotherapy in vitro and in vivo.

\section{MATERIALS AND METHODS}

Cell lines, antibodies and other reagents

REH and K562 cells were obtained from ATCC (Manassas, VA, USA) and propagated in RMPI-1640, $10 \%$ fetal bovine serum, $1 \%$ L-glutamine and $1 \%$ penicillin-streptomycin in a humidified incubator at $37^{\circ} \mathrm{C}$ and $5 \% \mathrm{CO}_{2}$ (Life Technologies, Carlsbad, CA, USA). In addition, medium for Sicontrol or SiPKR cells contained $2 \mu \mathrm{g} / \mathrm{ml}$ puromycin (Sigma-Aldrich, St Louis,

Department of Medicine, Division of Hematology and Oncology and the University of Florida Shands Cancer Center, University of Florida, Gainesville, FL, USA. Correspondence: Dr W Stratford May, Department of Medicine, Division of Hematology and Oncology, University of Florida, 2033 Mowry Road, Box 103633, Gainesville, FL 32610, USA.

E-mail: smay@ufl.edu

${ }^{1}$ These authors contributed equally to this work.

Received 4 July 2013; revised 21 July 2013; accepted 24 July 2013 
MO, USA) and medium for Sicontrol or SiPKR cells carrying luciferase contains both $2 \mu \mathrm{g} / \mathrm{ml}$ puromycin and $200 \mu \mathrm{g} / \mathrm{ml}$ hygromycin. DOX and PKR inhibitor compound (PKRI) were from Calbiochem/EMD Millipore (Darmstadt, Germany). FTY720 was from Cayman Chemical Company (Ann Arbor, MI, USA) and okadaic acid was from Millipore (Billerica, MA, USA). Hydrogen peroxide was from Sigma-Aldrich. Phospho-threonine 451-specific PKR rabbit polyclonal antibody was from Invitrogen/Biosource (Grand Island, NY, USA). PKR M02 monoclonal antibody clone 1D11 and $\mathrm{Bcl}-2$ antibody were from Abnova (Taipei City, Taiwan). Phospho-serine 51-specific elF $2 \alpha$, elF $2 \alpha$, phosphor-serine 70-specific BCl-2, BAX antibodies were from Cell Signaling Technology (Beverly, MA, USA). Actin Antibody was from Santa Cruz Biotechnology Inc. (Santa Cruz, CA, USA). Luciferase vector pGL 4.50 was from Promega (Madison, WI, USA).

\section{Inhibition of PKR expression by siRNA}

Transduction-ready lentivirus particles containing small interfering RNAs (siRNAs) specific for human PKR were used to knockdown PKR expression in REH and K562 cells according to the manufacturer's protocol (Santa Cruz Biotechnology, Inc., sc-36263). A green fluorescent protein-expressing lentivirus was used to measure transduction efficiency and optimize conditions. After transduction, stable cell lines were selected with $2 \mu \mathrm{g} / \mathrm{m}$ puromycin. Efficiency of knockdown was evaluated by western blotting.

\section{Cell apoptosis and survival assay}

Cells were treated with varying doses and times of DOX or $\mathrm{H}_{2} \mathrm{O}_{2}$, and apoptosis analyzed by annexin $\mathrm{V}$ staining using an annexin V-phycoerythrin (PE) apoptosis detection kit I according to the manufacturer's protocol (BD Biosciences, San Jose, CA, USA). Stained cells were analyzed on a FACSCalibur flow cytometer (BD Biosciences), placing the PE signal in FL2 and the 7-aminoactinomycin D (7-AAD) signals in FL3. Intact cells were gated to exclude small debris. Cells in the lower right quadrant of the FL2/FL3 dot plot (labeled with annexin V-PE only) were considered to be in early apoptosis and cells in the upper right quadrant (labeled with annexin V-PE and 7-AAD) in late apoptosis/necrosis. Cell survival was counted using Trypan blue dye exclusion assay.

\section{Cell invasion assay}

Cell invasion assay kit (Cat no. CBA110) from Cell Biolabs, Inc. (San Diego, (A, USA) was used. Briefly, $300 \mu \mathrm{l}$ cell suspension at the concentration of $0.5 \times 10^{6} / \mathrm{ml}$ in serum-free media was added to the upper chamber and $500 \mu \mathrm{l}$ media containing $10 \%$ fetal bovine serum was added to lower chamber. Cells were incubated for $24 \mathrm{~h}$ at $37^{\circ} \mathrm{C}$ and cells that invaded across the basement membrane were collected by incubating with extraction solution and stained per manufacturer's protocol. Cell number was determined by measuring $\mathrm{OD}_{560}$ in a plate reader.

\section{Immunoblotting and co-immunoprecipitation}

Cells were harvested, washed with phosphate buffered saline (PBS), lysed with detergent buffer and centrifugated. Then, $30 \mu \mathrm{g}$ of lysate was subjected to sodium dodecyl sulfate polyacrylamide gel electrophoresis and western blotting using the indicated antibodies. To perform coimmunoprecipitation, washed cells were lysed with $1 \times$ CHAPS buffer. Lysate $(500 \mu \mathrm{g})$ was precleared with nonspecific mouse antibody and Dynabead protein $G$ and then incubated with Dynabead protein G-conjugated antibody overnight. Dynabeads were collected magnetically, washed, resuspended in loading buffer and immunoblotted as indicated.

\section{Measurement of PP2A activity}

PP2A activity was measured using a PP2A phosphatase assay (Millipore). Briefly, cells were homogenized by dounce homogenizer, PP2A immunoprecipitated, incubated with phosphopeptide and phosphatase activity determined by measuring free phosphate in solution. PP2A activity (pM phosphate per $\mu \mathrm{g}$ protein) is presented as percentage of control.

\section{Isolation of mitochondria and alkaline treatment}

Mitochondria were isolated using a mitochondria isolation kit (Miltenyi Biotech, Auburn, CA, USA). Briefly, cells were washed, lysed and incubated with $50 \mu \mathrm{l}$ Anti-TOM22 Microbeads. Labeled mitochondria were isolated using a MACS Midi (Miltenyi Biotech) column and eluted with $1 \times$ separation buffer. Isolated mitochondria were treated with an alkaline buffer ( $300 \mathrm{~mm}$ sucrose, $0.1 \mathrm{Na}_{2} \mathrm{CO}_{3}, \mathrm{pH} 11.3$ ) for $30 \mathrm{~min}$ at $4{ }^{\circ} \mathrm{C}$ to dissipate any protein not integrally part of mitochondria, followed by centrifugation for $15 \mathrm{~min}$ at $80000 \times g$ at $4{ }^{\circ} \mathrm{C}$. The pellet was collected and proteins analyzed by sodium dodecyl sulfate polyacrylamide gel electrophoresis and immunoblotting. Purity of mitochondria isolated was determined using a COX (Cell Signaling Technology) antibody to identify intramitochondrial contents and an HSP90 antibody (Santa Cruz Biotechnology Inc.) to detect cytoplasmic contaminants.

\section{Xenograft studies}

All studies were approved by the University of Florida institutional animal care and use committee. On the day of tumor inoculation, NOD/SCID/IL2r$\gamma$ null (NSG) mice received 200 cGy of total body irradiation at a dose rate of $325 \mathrm{cGy} / \mathrm{min}$. Immediately following irradiation, 10 million REH Sicontrol or REH SiPKR cells suspended in $100 \mu \mathrm{l}$ RPMI- 1640 were injected through tail vein. For 3 days before irradiation and 14 days post irradiation, animals were given SulfaTrim antibiotic water. In the treatment group, mice were given a single dose of DOX $(5 \mathrm{mg} / \mathrm{kg})$ intravenously 3 days after inoculation. In the placebo group, mice were given equivalent volume of normal saline. In the group treated with both DOX and FTY720, FTY720 was given at $2 \mathrm{mg} / \mathrm{kg} /$ day intraperitoneally from day 1 to day 14 following irradiation. After inoculation of leukemia cells, mice were evaluated daily by veterinary staff and mice with a body condition score of $\leqslant 2$ were killed. ${ }^{29}$

Starting 2 weeks post inoculation, tumor burden was monitored weekly using an IVIS Lumina II In Vivo Imaging System (Xenogen, Alameda, CA USA). On the day of imaging, mice were given $150 \mathrm{mg} / \mathrm{kg}$ D-luciferin intraperitoneally $10 \mathrm{~min}$ before imaging, anesthetized with isoflurane and subjected to imaging.

At 6 weeks after irradiation, moribund mice were killed and bone marrow harvested from femurs. Cells were stained with anti-human CD45-PE/anti-mouse CD45 FITC (eBioscience, San Diego, CA, USA) and the percentage of human CD45 + mouse CD45 - cells was measured by flow cytometry or subjected to western blotting for detection of PKR levels.

\section{Statistics}

All data are presented as the mean \pm s.d. Significant differences were determined by $t$-test using SPSS Statistics Premium software (IBM, Armonk, NY, USA). Kaplan-Meier curve of overall survival of mice was plotted and analyzed by Graphpad Prism 6 software (Graphpad software, La Jolla CA, USA). The criterion for a statistical significance is $P<0.05$.

\section{RESULTS}

Loss of PKR inhibits apoptosis and promotes survival in response to cellular stress

To test the role of PKR on human leukemic cell growth, both lymphoid REH and myeloid K562 leukemic cells were transduced with lentivirus containing either a PKR SiRNA (SiPKR) or a control scrambled sequence SiRNA (Sicontrol). Importantly, similar transduction and selection efficiencies were observed using either sIPKR or siControl-expressing lentivirus (data not shown). Significant (>90\%) reduction of PKR expression in siPKR compared with siControl cells was confirmed by western blot (Figure 1a). Importantly, cell proliferation rates were unaffected by PKR knockdown under normal (nonstress) conditions (Figure 1b). However, under nutrient deprivation stress conditions, both REH and K562 cells with knockdown PKR demonstrate increased invasion rates across an artificial basement membrane (Figures $1 \mathrm{c}$ and d). This suggests that the proapoptotic function of PKR under the stress of growth factor deprivation conditions may also decrease cell invasion.

To better characterize the role of PKR on cell survival during stress, cells were exposed to increasing concentrations of either $\mathrm{H}_{2} \mathrm{O}_{2}$ or the antileukemic agent, DOX for $48 \mathrm{~h}$. Cell survival and apoptosis was measured by annexin V staining. Significantly, reduced PKR expression in both $\mathrm{K} 562$ and REH cells delays the rate and extent of apoptosis (Figure 1e). For instance, following treatment of REH cells with $200 \mu \mathrm{M} \mathrm{H}_{2} \mathrm{O}_{2}$ for $48 \mathrm{~h}$, SiPKR cells have a $33 \%$ decrease in apoptosis $(P<0.01)$ compared with control cells (Figure 1e). In addition, PKR knockdown cells exposed to $\mathrm{H}_{2} \mathrm{O}_{2}$ for 
a

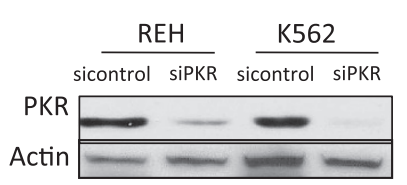

C

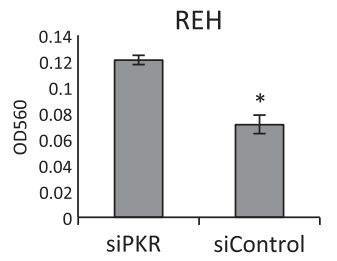

e

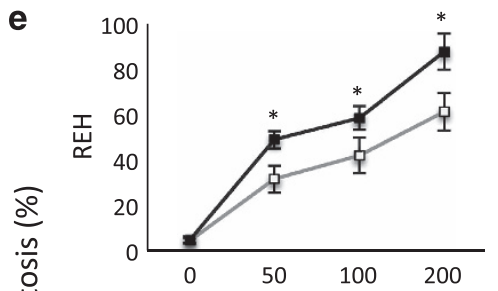

b 5

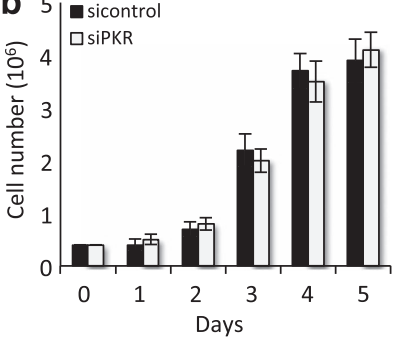

d
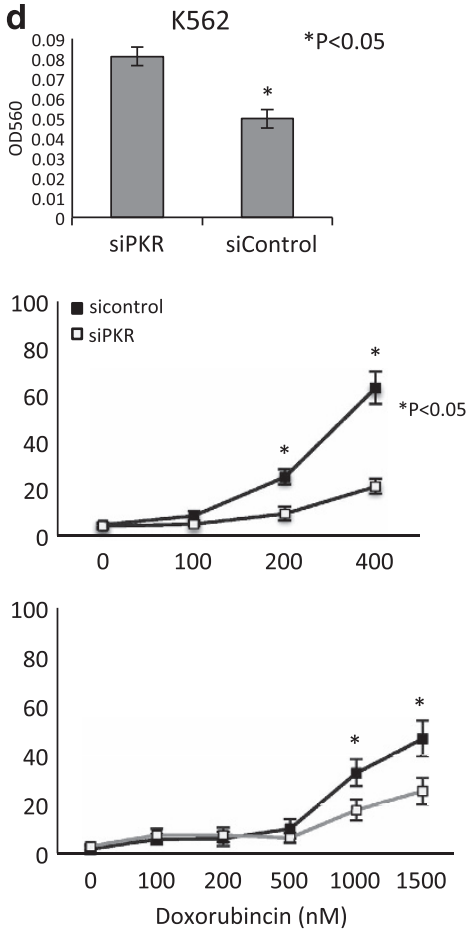

f

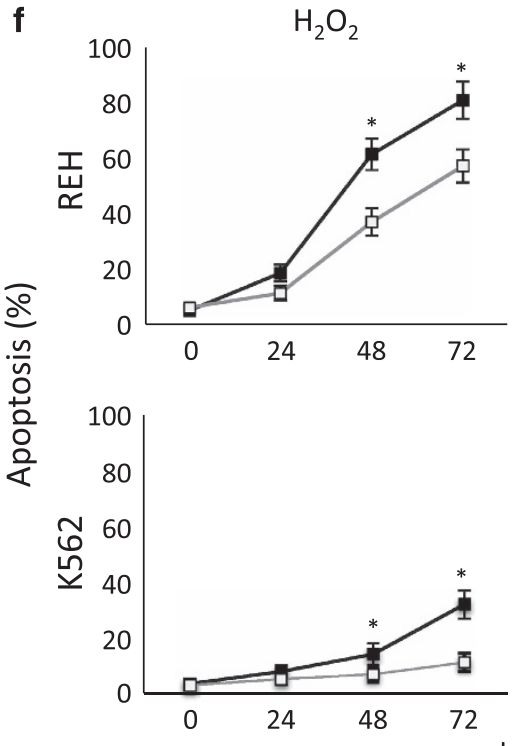

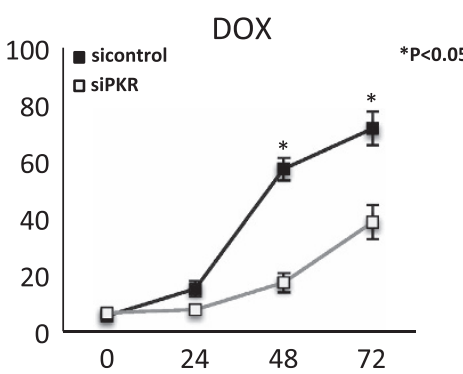

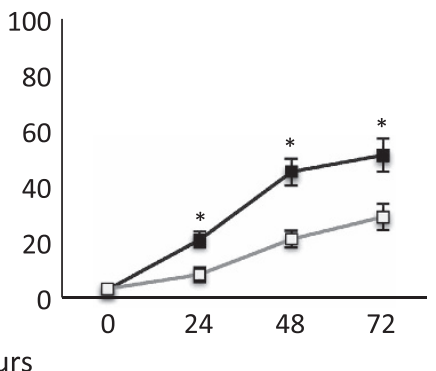

Figure 1. Loss of PKR inhibits apoptosis and promotes survival in response to cellular stress. (a) REH and K562 cells were transduced with lentivirus expressing either PKR SiRNA (SiPKR) or scrambled control SiRNA (Sicontrol). Knockdown of PKR expression was confirmed by western blot. (b) $0.4 \times 10^{6}$ of both Sicontrol and SiPKR cells were cultured for 5 days and cell numbers were counted daily. (c, d) REH or K562 cells were subjected to transwell assay. Invasion across basement membrane was analyzed. (e) Cells were exposed to various concentrations of $\mathrm{H}_{2} \mathrm{O}_{2}$ or DOX for $48 \mathrm{~h}$ and apoptosis evaluated by flow cytometry using annexin V-PE and 7-AAD staining. (f) REH cells were treated with either $100 \mu \mathrm{m} \mathrm{H} \mathrm{O}_{2}$ or $400 \mathrm{~nm}$ DOX, and $\mathrm{K} 562$ cells were exposed to $400 \mu \mathrm{m} \mathrm{H}_{2} \mathrm{O}_{2}$ or $1500 \mathrm{~nm}$ DOX. Apoptosis assays were performed at designated time points $\left(0,24,48\right.$ and 72 h) by flow cytometry. All results are representatives of three independent experiments. ${ }^{*}$ Indicates $P<0.05$. 
up to $72 \mathrm{~h}$ demonstrate a $20-25 \%$ decrease in apoptosis compared with control cells (Figure 1f). Similarly, PKR knockdown cells treated with DOX for $72 \mathrm{~h}$ have a 33 and $22 \%$ decrease in apoptosis compared with Sicontrol cells (Figure 1f). These results indicate that PKR is required for the full and potent effect of an oxidative or chemotherapeutic stress application that can initiate apoptosis.

Suppression of PKR inhibits PP2A activity and increases Bcl-2 phosphorylation and resistance to apoptosis

As decreased PKR expression inhibits stress-induced apoptosis, we examined the mechanism by which PKR may regulate apoptosis in leukemic cell lines. ${ }^{6}$ Significantly, although $\mathrm{Bcl}-2$ expression is not affected by reduced $\mathrm{PKR}, \mathrm{BCl}-2$ phosphorylation at serine 70 (p-S70-Bcl-2) is significantly increased, indicating one mechanism by which loss of PKR may enhance cell survival (Figure 2a).

As we previously reported that the protein phosphatase PP2A is a direct negative regulator of $\mathrm{BCl}-2$, we measured PP2A activity in cells with reduced PKR or treated with a specific small-molecule PKR inhibitor (PKRI). ${ }^{30}$ Importantly, PP2A activity is significantly decreased to $\sim 60 \%$ of control cells in cells with reduced PKR whereas the level of PP2A/C expression does not change (Figures $2 \mathrm{a}$ and $\mathrm{b}$ ). Furthermore, when cells are treated with increasing concentrations of PKRI, phospho-PKR (a measure of enzymatic activation), phosphorylation of the PKR substrate elF2 $\alpha$ and PP2A activity are decreased, confirming their upstream regulation by PKR (Figure $2 \mathrm{~d}$ ). In addition, although total PP2A or $\mathrm{BCl}-2$ expression is not altered, $\mathrm{BCl}-2$ phosphorylation is markedly increased upon treatment with PKRI (Figure 2c). These results indicate that PKR catalytic activity is required to activate PP2A that results in dephosphorylation of $\mathrm{Bcl}-2$. This may explain, at least in part, why cells with reduced PKR activity display enhanced survival.

Activation of PP2A restores chemosensitivity to cells expressing decreased PKR

As PKR-dependent PP2A activation may be necessary for the full and potent initiation of apoptosis, we investigated whether direct activation of PP2A can restore sensitivity of cells with decreased PKR to DOX or $\mathrm{H}_{2} \mathrm{O}_{2}$. For these studies, the specific PP2A activator FTY720 that has been reported to have antileukemic activity in vitro and in vivo was tested. ${ }^{31-35}$ As a control, we used the PP2A inhibitor, okadaic acid..$^{36}$ Importantly, we tested various concentrations of FTY720, to determine an optimum concentration $(2.5 \mu \mathrm{m})$ that increases PP2A activity by approximately $50 \%$ but does not affect REH or K562 cell viability under normal growth conditions for use in subsequent studies (data not shown and Figure 3a). As expected, results demonstrate that treatment of cells with FTY720 decreases, whereas treatment with okadaic acid increases $\mathrm{Bcl}-2$ phosphorylation (Figure $3 \mathrm{~b}$ ). Significantly, FTY720 treatment of PKR knock-down cells restores $\mathrm{H}_{2} \mathrm{O}_{2}$ and DOX sensitivity to levels comparable with siControl cell (Figures $3 \mathrm{c}$ and $\mathrm{d}$ ).

Loss of PKR stabilizes Bcl-2/Bax association and inhibits Bax insertion into the OMM

As PKR-dependent PP2A activation may be required for $\mathrm{BCl}-2$ dephosphorylation, which induces apoptosis, we investigated

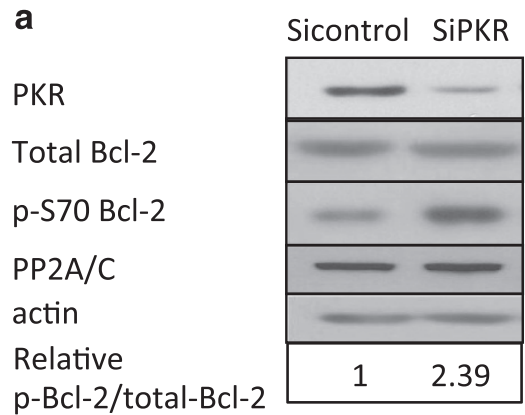

C

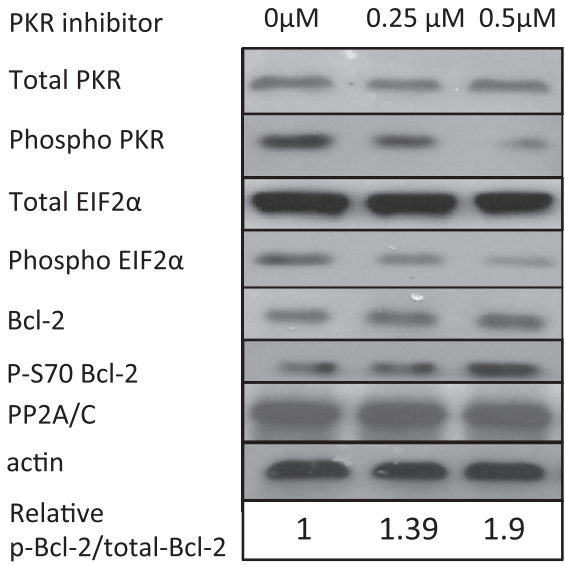

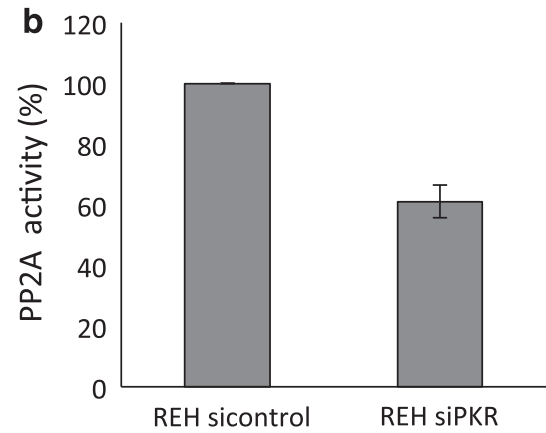

d

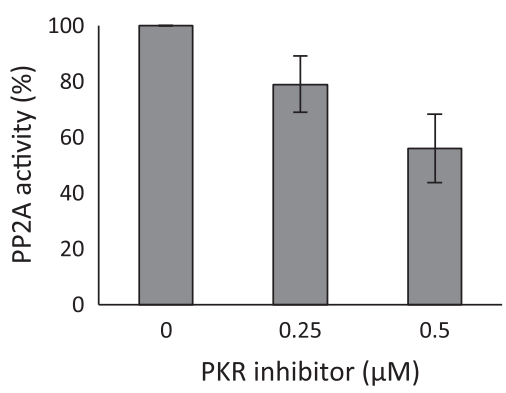

Figure 2. Inhibition of PKR activity leads to decreased PP2A activity and increased phosphorylation of Bcl-2. (a) Western blot to evaluate protein levels of PKR, total Bcl-2, phosphor Bcl-2 (S70) in REH Sicontrol vs SiPKR cells. (b) PP2A activity in Sicontrol and SiPKR cells was measured by in vitro assay. (c) REH cells were pretreated with various concentrations of PKR inhibitor for $12 \mathrm{~h}$ before being lysed and subjected to western blot to evaluate protein levels of total PKR, phosphor PKR (T451), total EIF2 $\alpha$, phosphor EIF2 $\alpha$ (S51), total Bcl-2 and phosphor Bcl-2 (S70). (d) PP2A activity assay was performed to evaluate PP2A activity in PKRI-treated cells. PP2A activity is presented as percentage of control, and representative results from three independent experiments are shown. 
a

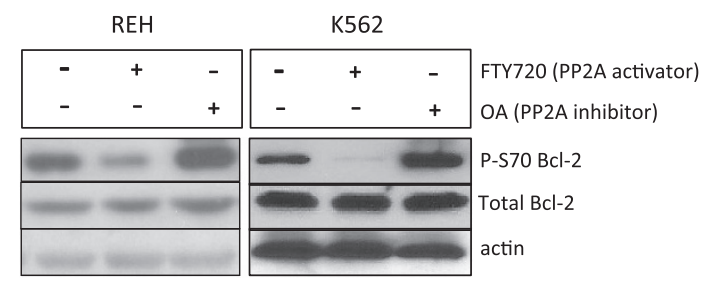

b

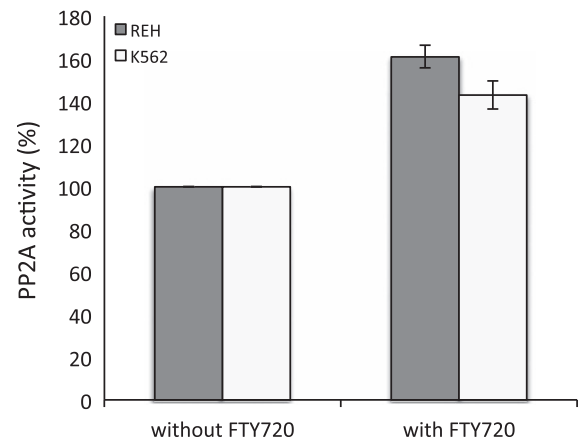

C

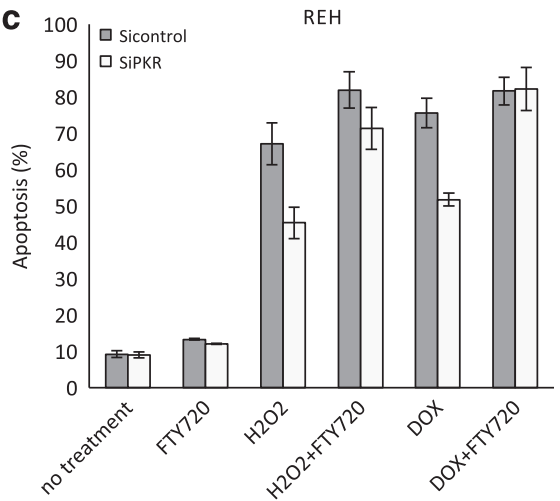

d

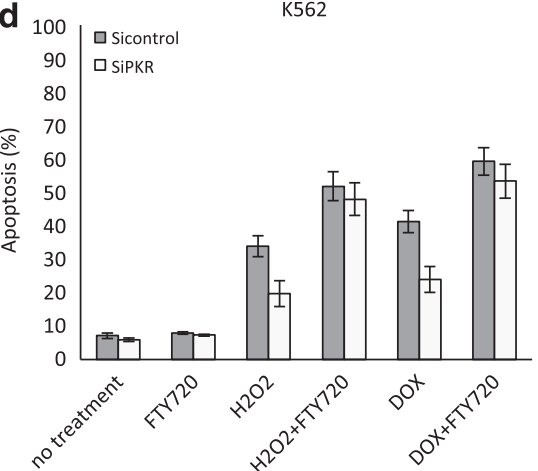

Figure 3. Modulation of PP2A activity affects Bcl-2 activity and cell apoptosis in REH cells. (a) REH or K562 cells were treated with $2.5 \mu \mathrm{M}$ FTY720 for $12 \mathrm{~h}$, and PP2A activity was evaluated. (b) REH cells were treated with either $1 \mathrm{~nm}$ okadaic acid or $2.5 \mu \mathrm{m}$ FTY720 for $12 \mathrm{~h}$ and subjected to western blot for evaluation of total $\mathrm{Bcl}-2$ and p-Bcl-2 levels. (c) REH cells were pretreated with either $2.5 \mu \mathrm{M}$ FTY720 or dimethyl sulfoxide (DMSO) for $12 \mathrm{~h}$, and then exposed to either $100 \mu \mathrm{m} \mathrm{H}_{2} \mathrm{O}_{2}$ or $600 \mathrm{~nm}$ DOX for $48 \mathrm{~h}$. Cells were stained by annexin $\mathrm{V}$ and apoptosis was analyzed by flow cytometry. (d) K562 cells were pretreated with either $2.5 \mu \mathrm{M}$ FTY720 or DMSO for $12 \mathrm{~h}$, and then exposed to either $800 \mu \mathrm{m} \mathrm{H}_{2} \mathrm{O}_{2}$ or $1500 \mathrm{~nm}$ DOX for $48 \mathrm{~h}$. Cells were stained by annexin V and apoptosis was analyzed by flow cytometry.

whether PKR may affect the function of $\mathrm{Bcl}-2$ to associate with Bax and regulate the proapoptotic function of Bax of insertion into the OMM. Following treatment with $\mathrm{H}_{2} \mathrm{O}_{2}$, the $\mathrm{Bcl}-2$ /Bax complex was reciprocally co-immunoprecipitated from cells that express either a SiPKR or control siRNA. Significantly, in both REH and K562 cells, reduced PKR expression was discovered to increase the association of $\mathrm{Bcl}-2$ and $\mathrm{Bax}$ following treatment with $\mathrm{H}_{2} \mathrm{O}_{2}$ (Figures $4 a$ and b).

Next, we isolated whole mitochondria from cells following treatment with $\mathrm{H}_{2} \mathrm{O}_{2}$ to measure the level of Bax insertion into the OMM. In either Sicontrol REH or K562 cells, Bax insertion into the OMM increases in a dose-dependent manner upon treatment with $\mathrm{H}_{2} \mathrm{O}_{2}$ (Figures $4 \mathrm{c}$ and d). In contrast, cells with decreased PKR expression display a significantly reduced level of Bax insertion into the OMM following treatment (Figures $4 c$ and $d$ ). These findings strongly suggest that loss of PKR expression/activity inhibits and delays apoptosis in response to oxidative stress by stabilizing the Bcl-2/Bax complex, resulting in decreased Bax OMM insertion following stress.

Decreased PKR enhances the extent and chemoresistance of leukemia in vivo

To test whether the loss of PKR expression affects leukemia cell spread and survival in vivo, we monitored leukemic tumor load in a mouse xenograft model and compared results with mouse survival. NSG mice were engrafted with either $1 \times 10^{6}$ SiPKR or Sicontrol cells that express a luciferase reporter. The Sicontrol and SiPKR cells used were confirmed to have comparable bioluminescence signals (data not shown). Following engraftment, imaging of mice was performed weekly and to quantify leukemia tumor burden (Figure 5a). Significantly, xenografted mice receiving SiPKR cells display an increased rate of tumor spread compared with Sicontrol xenografts (Figure 5b). For example at 4 weeks following inoculation, mice receiving siPKR cells display approximately twofold more leukemic cell burden than mice receiving Sicontrol cells (Figure $5 b$ ).

Next, we examined whether reduced expression of PKR in xenografted leukemic cells may affect the efficacy of DOX treatment in vivo. Briefly, xenografts were established for 3 days at which time mice received a single intravenous dose of either vehicle or $5 \mathrm{mg} / \mathrm{kg}$ DOX. The rate of tumor spread was compared to evaluate the response to treatment. Significantly, xenografts with SiPKR cells were less sensitive to DOX treatment in contrast to xenografts of Sicontrol cells. Importantly, after 4 weeks, DOXtreated Sicontrol xenografts displayed an $\sim 90 \%$ reduction of tumor burden as compared with vehicle-only-treated mice. In contrast, treatment of mice engrafted with SiPKR cells results in only $\sim 50 \%$ reduction of tumor burden (Figure $5 \mathrm{c}$ ). Thus, decreased PKR expression in engrafted leukemic cells results in an almost twofold reduction in sensitivity to a single treatment with DOX in vivo.

Finally, we tested whether activation of the PKR/PP2A signaling axis may restore sensitivity to chemotherapy of mice xenografted with cells that have reduced PKR expression. Briefly, 3 days following engraftment, mice were administered a single dose of DOX as above followed by daily injection with FTY720 at $2 \mathrm{mg} / \mathrm{kg}$ for 2 weeks. Mice that received both DOX and FTY720 displayed a significantly decreased leukemia cell burden compared with mice treated with DOX alone (Figure 5c). Thus, co-treatment with FTY720 and DOX results in an $\sim 50 \%$ decrease in tumor volume at 3 weeks compared with mice treated with DOX alone (Figure $5 \mathrm{c}$ ).

The overall survival of mice in each group was evaluated by Kaplan-Meier analysis (Figure 5d). Consistent with increasing 


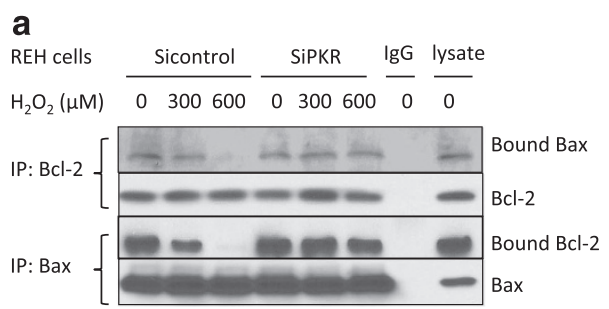

C

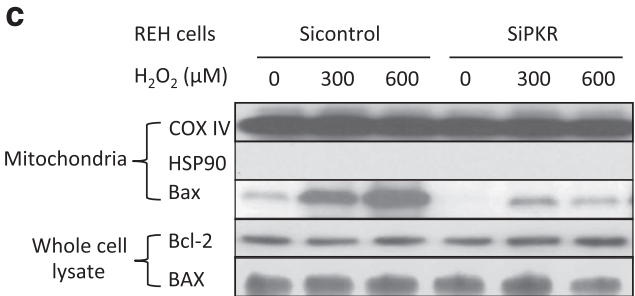

b

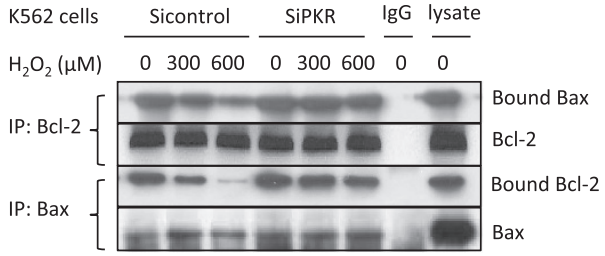

d

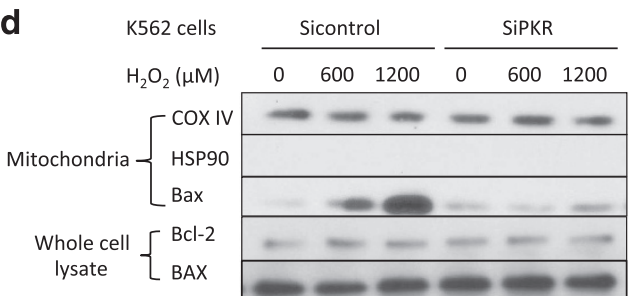

Figure 4. Loss of PKR stabilizes $\mathrm{Bcl}-2 / \mathrm{Bax}$ binding and inhibits Bax insertion to OMM. (a) REH or (b) $\mathrm{K} 562$ cells were exposed to $\mathrm{H}_{2} \mathrm{O}_{2}$ for $6 \mathrm{~h}$, lysed and co-immunoprecipitation of $\mathrm{Bcl}-2$ and Bax performed. (c) REH cells were treated with $\mathrm{H}_{2} \mathrm{O}_{2}$ for $6 \mathrm{~h}$. Mitochondria were isolated and treated with alkaline buffer. Mitochondrial debris was then collected by ultracentrifugation and subject to western blot for evaluating BAX level. (d) $\mathrm{K} 562$ cells were treated with $\mathrm{H}_{2} \mathrm{O}_{2}$ for $6 \mathrm{~h}$. Bax insertion was evaluated as described previously. Total cellular levels of Bax and Bcl-2 after 6-h exposure to $\mathrm{H}_{2} \mathrm{O}_{2}$ were evaluated by western blot.

tumor burden in mice xenografted with SiPKR cells, these mice also have significantly shorter survival compared with mice inoculated with Sicontrol cells $(P=0.0042)$. Furthermore, although the survival of mice in both groups could be prolonged following a single treatment with DOX, survival is significantly increased for mice engrafted with Sicontrol cells compared with those engrafted with PKR knockdown leukemic cells $(P=0.0021$ vs $P=0.1037$ ). Importantly, survival of SiPKR engrafted mice following treatment with the combination of FTY720 and DOX is also significantly increased over DOX-only-treated mice $(P=0.0164)$. This suggests that reactivation of the PKR/PP2A pathway in leukemic cells displaying reduced PKR may restore sensitivity to antileukemic treatment.

To confirm that inoculated leukemia cells did engraft bone marrow (the pathological initiation site of leukemia development), both human and mouse CD45 + cells were quantified after isolation from bone marrow of xenografted mice. Importantly, all moribund mice analyzed displayed over 90\% human CD45 + leukemia cells in their bone marrow (Figure 5e). In addition, western blot analysis of bone marrow cells isolated from moribund mice confirmed that the REH SiPKR leukemic cells continued to display reduced expression of PKR at the time of death (Figure $5 f$ ).

\section{DISCUSSION}

Our findings demonstrate that activity of the interferon-inducible double-stranded RNA-dependent protein kinase PKR may inhibit human leukemic cell growth and promote sensitivity to chemotherapy or other stress applications including direct oxidative stress. Significantly, K562 or REH cells with reduced PKR expression or those treated with PKRI display decreased substrate phosphorylation of elF $2 \alpha$, decreased PP2A activity, increased Bcl-2 phosphorylation and reduced apoptosis following treatment with either DOX or $\mathrm{H}_{2} \mathrm{O}_{2}$. In addition, inhibition of PKR activity promotes $\mathrm{BCl}-2 / \mathrm{BAX}$ association and inhibits $\mathrm{BAX}$ insertion into the OMM following stress that triggers the mitochondrial dysfunction observed with activation of the intrinsic apoptotic pathway. These results both confirm and extend our previous report that the anti-apoptotic activity of $\mathrm{Bcl}-2$ is enhanced by phosphorylation and that dysregulation of the mitochondria is inhibited by phosphorylation of $\mathrm{Bcl} 2$ that leads to reduced Bax insertion into the OMM. ${ }^{37}$ Significantly, and of potential clinical relevance, we find for the first time that mice xenografted with leukemia cells that have reduced PKR display an increased rate of tumor spread compared with control xenografts, and treatment with FTY720, a PP2A activator, can rescue this phenotype. In addition, increased chemosensitivity of FTY70-treated cells occurs in close association with decreased $\mathrm{Bcl}-2$ phosphorylation and enhanced apoptosis. Taken together, these data indicate that decreased PKR activity in human acute leukemia cells may inhibit the initiation of apoptosis and promote leukemogenic survival by a mechanism dependent, at least in part, on PP2A activation and $\mathrm{BCl}-2$ dephosphorylation. This notion is further supported by our preliminary results using Reverse Phase Protein Array (RPPA) analysis of CD34 + leukemic cells from 511 acute myeloid leukemia patient samples that indicate that PKR expression and activity is significantly decreased in acute myeloid leukemia blast cells compared with the CD34+ cells from healthy donors (RL Bennett, SM Kornblau and WS May; data not shown). Thus, decreased PKR expression and activity may be a common theme in acute leukemia and, if this is the case, may point the way to novel therapeutic strategies for such patients.

Importantly, we found that loss of PKR expression potentiates leukemia cell survival following chemotherapy treatment in vivo. Recently, we reported that PKR-null mice and mice expressing a catalytically null, dominant-negative PKR transgene (specifically in hematopoietic cells) have increased numbers of hematopoietic stem/progenitor cells in association with increased peripheral blood cell counts as the mice age. ${ }^{6}$ Significantly, the loss of PKR activity in bone marrow cells from these mice promoted an increased frequency of hematopoietic stem/progenitor cells as evidenced by more robust hematopoietic colony formation and enhanced resistance to apoptosis following hematopoietic growth factor deprivation, treatment with inflammatory cytokines or irradiation. ${ }^{6}$ Now, findings here extend these results by demonstrating that xenografts of REH acute lymphoblastic leukemia cells with reduced PKR display a significantly greater rate of engraftment and reduced overall survival than control cell xenografts. Significantly, tumor volume following 4 weeks of engraftment is more than double in mice engrafted with REHSiPKR cells versus SiControl cells, and SiPKR xenografts are more resistant to DOX treatment compared with SiControl xenografts. Together, these results lead us to propose that downregulation of 
PKR activity in xenografted cells may promote leukemogenesis and chemoresistance because of reduced sensitivity of these cells to proapoptotic stress such as growth factor deprivation or exposure to inflammatory cytokines. In support of this and consistent with the in vitro results, sensitivity of SiPKR xenografts to DOX can be 'rescued' by treatment with the PP2A activator,

a

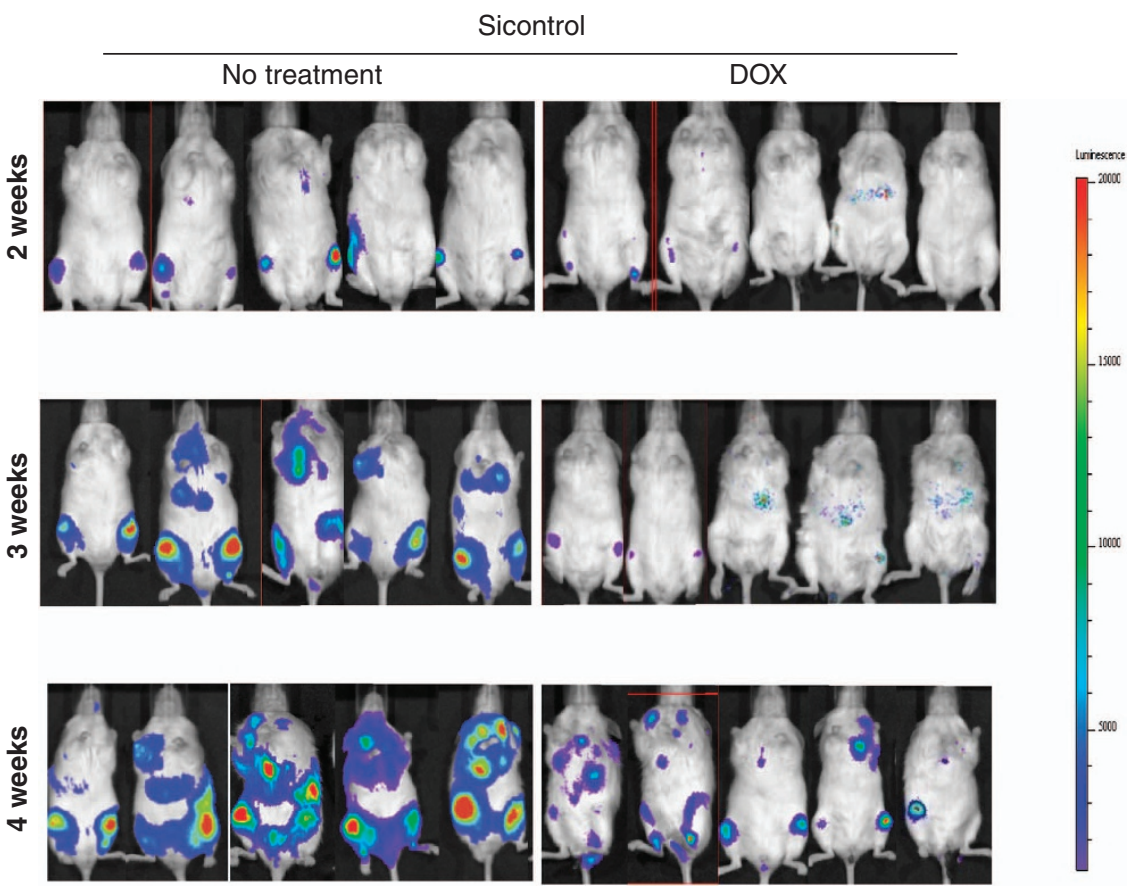

SiPKR
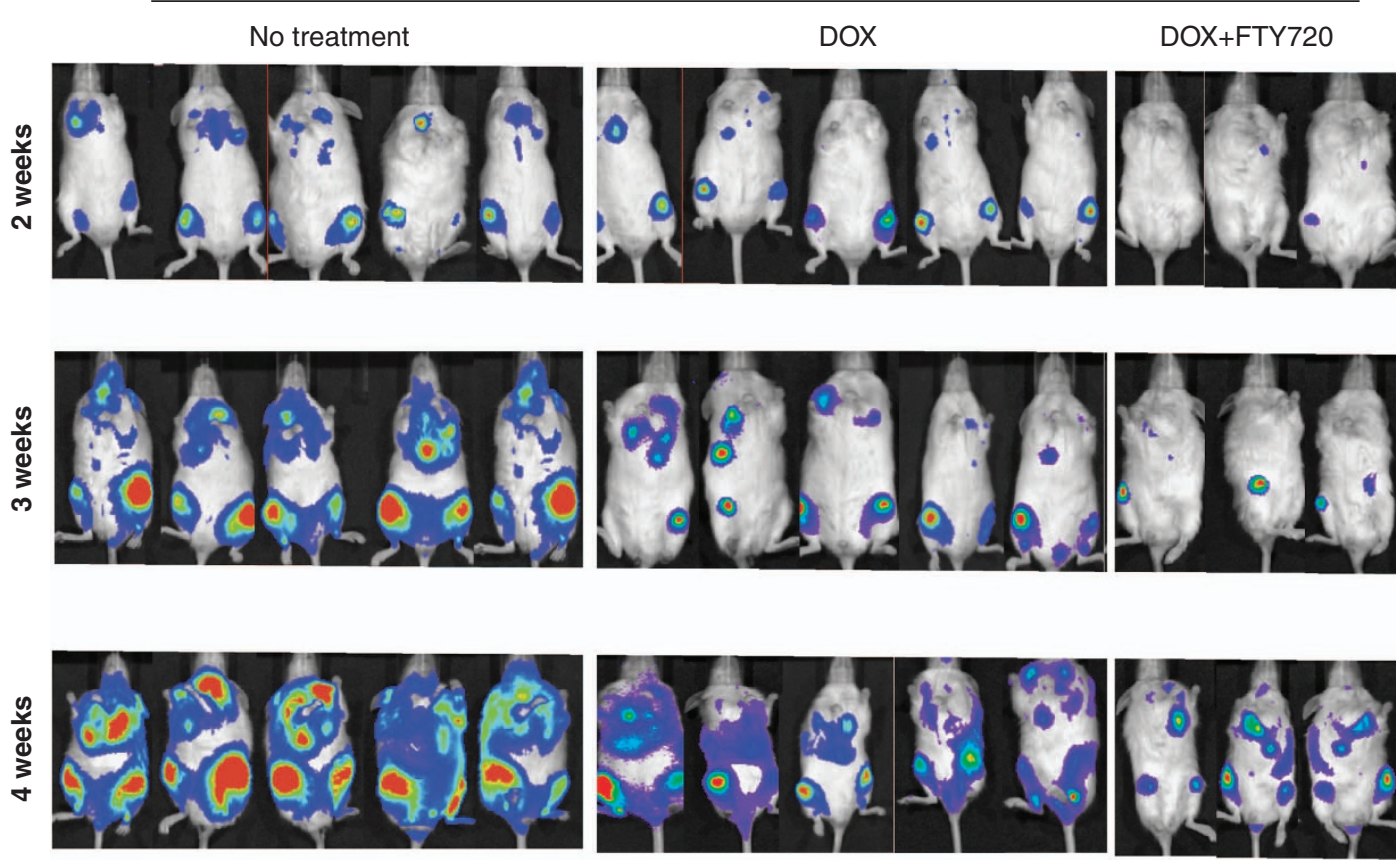

Figure 5. In leukemia xenografts, reduced expression of PKR promotes tumor progression and chemoresistance. Reactivation of the PKR/PP2A signaling axis by FTY720 treatment rescues chemosensitivity. (a) NSG mice were divided into five experiment groups (Sicontrol no treatment, Sicontrol DOX treatment, SiPKR no treatment, SiPKR DOX treatment and SiPKR DOX + FTY720 treatment) and inoculated with REH cells (Sicontrol or SiPKR) carrying luciferase gene followed by corresponding intervention in each group. Tumor progression was monitored by weekly in vivo imaging. (b) Tumor progression is evaluated and compared in untreated groups between mice carrying REH Sicontrol cells and mice carrying REH SiPKR cells. (c) Tumor progression following $5 \mathrm{mg} / \mathrm{kg}$ DOX treatment (as described in the Materials and methods section) in mice carrying either Sicontrol or SiPKR cells is evaluated and compared. Mice engrafted with REH SiPKR cells were also treated with same dose of DOX plus $2 \mathrm{mg} / \mathrm{kg}$ daily FTY720 for 2 weeks, tumor progression was evaluated and compared with mice carrying same leukemic cells but treated with only DOX. (d) Kaplan-Meier curve of overall survival of mice in each group was plotted. (e) Bone marrow cells from moribund mice carrying leukemia were harvested and stained with both human and mouse CD45 antibody, and the results were analyzed by flow cytometry. (f) Bone marrow cells from moribund mice carrying leukemia were harvested and lysed. PKR expression was evaluated by western blot. *Indicates $P<0.05$. 

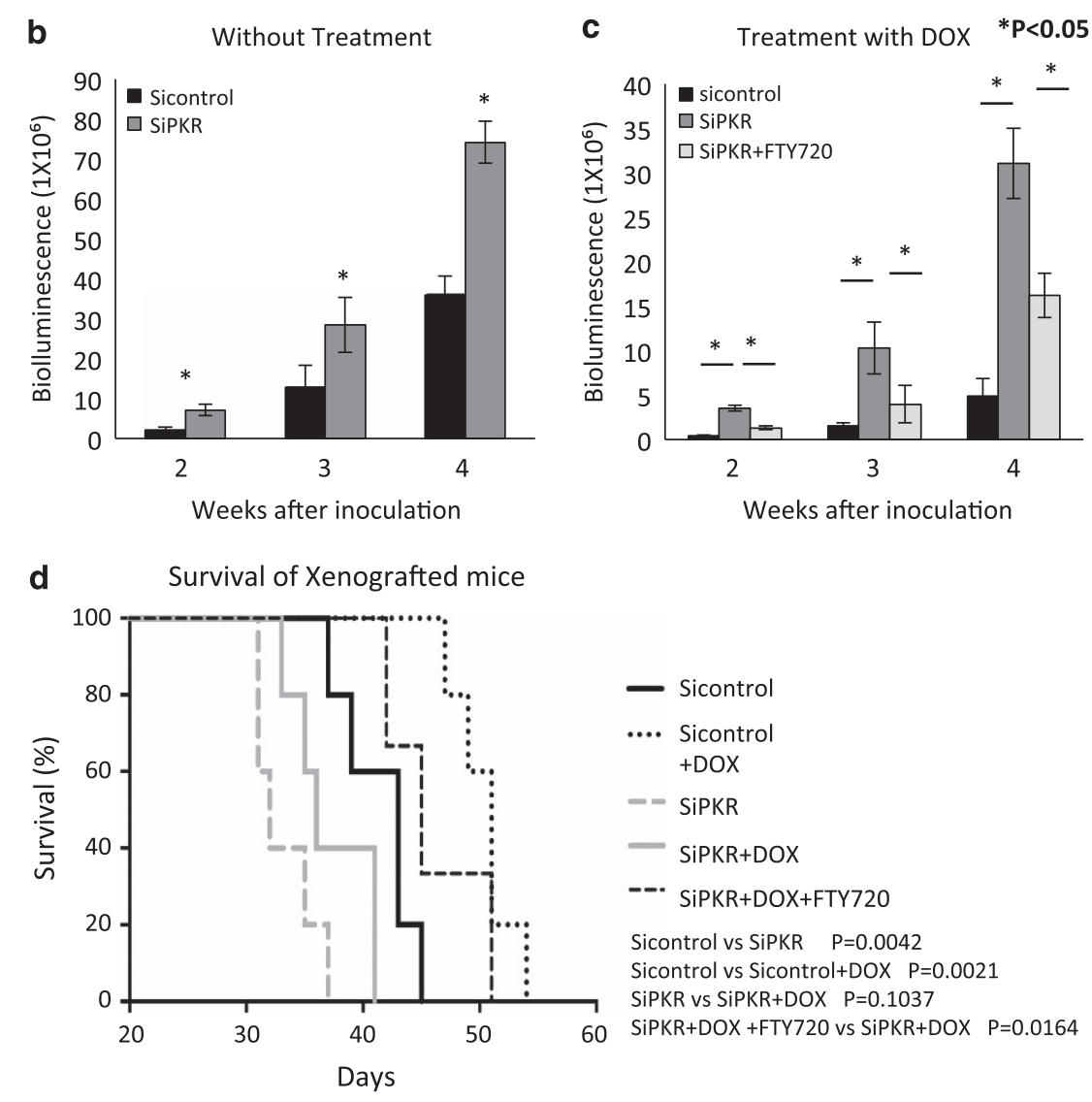

$$
\begin{array}{ll}
\text { - Sicontrol } \\
\ldots . & \text { Sicontrol } \\
& + \text { DOX } \\
- & \text { SiPKR } \\
- & \text { SiPKR+DOX } \\
-- & \text { SiPKR+DOX+FTY720 }
\end{array}
$$

Sicontrol vs SiPKR $P=0.0042$

Sicontrol vs Sicontrol+DOX $P=0.0021$

SiPKR VS SiPKR+DOX $P=0.1037$

e

Sicontrol
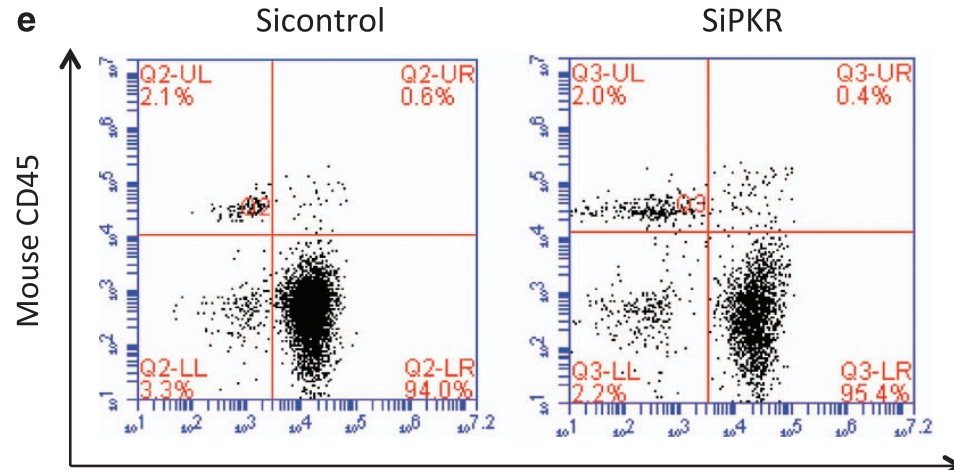

Human CD45

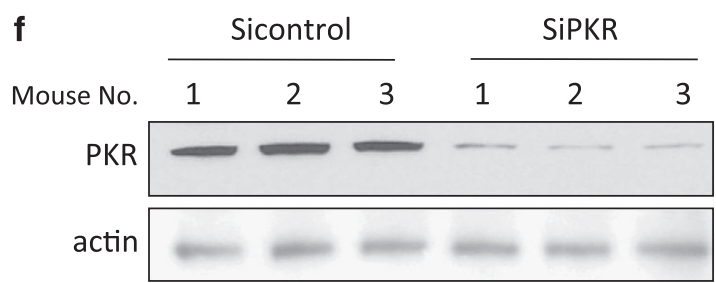

Figure 5. (Continued).

FTY720. Thus, reduced PP2A activation delays activation of the intrinsic mitochondria apoptotic mechanism and may, at least in part, account for the greater rate of engraftment observed for leukemic cells expressing reduced PKR.

Alternatively, our findings indicate that decreased PKR expression promotes cell invasion of hematologic cell lines in vitro that may contribute to the increased extent of engraftment and increased tumor volume displayed by REH siPKR cells. However, in preliminary studies, we did not detect any PKR-dependent changes in expression of genes important for cell invasion/ migration (data not show). Furthermore, our findings here and those previously published indicate that PKR is necessary and sufficient to initiate apoptosis in response to growth factor starvation. Thus, we believe we can attribute the effect of PKR on cell invasion to increased survival of cells with reduced PKR. Interestingly, we previously reported that decreased PKR inhibits 
invasion of breast cancer cell lines in vitro using the same assay, but these cells also display increased expression of proteins that inhibit or circumvent PKR activity. Thus, specific PKR-dependent antitumor mechanisms may vary based on cell type.

Potentially of clinical significance, the results demonstrate that reactivation of the PKR/PP2A axis in cells with reduced PKR activity by treatment with the PP2A activator FTY720 restores sensitivity of leukemic cells to DOX, an important standard of care treatment in induction of acute leukemia. Interestingly, FTY720 is a shingosine 1-phosphate (S1P) receptor modulator that has already been used in clinical trials to treat relapsing multiple sclerosis and to prevent organ rejection following renal transplantation. ${ }^{38,39}$ In addition, others have reported FTY720 as a potential antileukemic drug both in vitro and in vivo but the mechanism is not clear. Now, our results confirm that FTY720 may activate PP2A, which is frequently inhibited in hematopoietic malignancies. ${ }^{40}$ Significantly, as reduced PKR may frequently occur in acute leukemias and our findings here suggest that loss of PKR leads to reduced PP2A activity, a 'PP2A activation strategy' might be envisioned to therapeutically enhance leukemia therapy in patients whose tumors display reduced PKR activity. This notion is supported by our findings here that FTY720 more effectively enhances apoptosis induced by $\mathrm{H}_{2} \mathrm{O}_{2}$ or DOX in human leukemic cells displaying reduced PKR expression than control cells. Therefore, PKR expression/activity may serve as a predictor of effectiveness of FTY720 treatment. In addition, although previous studies test FTY720 alone as an antileukemia agent, our study for the first time combines it with DOX both in vitro and in vivo. In this manner, we were able to treat leukemic cells with a considerably lower dosage of FTY720 that stimulates PP2A activation but does not by itself significantly affect cell proliferation or survival. This indicates that FTY720 may be useful in combination with cytotoxic chemotherapy treatments like doxorubicin to facilitate their antileukemic effect while avoiding potential serious side effects from full-dose FTY720 treatment.

Taken together, our results demonstrate that loss of PKR function contributes to leukemia disease progression because of defects in the intrinsic apoptosis mechanism. In the future, loss of PKR activity may serve as a useful clinical predictor of chemoresponsiveness to DOX-containing regimens and/or sensitivity to FTY720. Importantly, reactivation of the PKR/PP2A signaling axis may serve as a novel therapeutic approach to enhance response to chemotherapy, especially in leukemia with low PKR activity.

\section{CONFLICT OF INTEREST}

The authors declare no conflict of interest.

\section{ACKNOWLEDGEMENTS}

We thank Tammy Flagg for technical assistance. This work was supported by NIH/NHLBI R01 HL054083.

\section{AUTHOR CONTRIBUTIONS}

$X C$ and RLB designed experiments, performed research and wrote the manuscript; $X L$ and MB performed experiments; and WSM designed experiments and wrote the manuscript.

\section{REFERENCES}

1 Ito T, Jagus R, May WS. Interleukin 3 stimulates protein synthesis by regulating double-stranded RNA-dependent protein kinase. Proc Natl Acad Sci USA 1994; 91: 7455-7459.

2 Bennett RL, Blalock WL, Abtahi DM, Pan Y, Moyer SA, WS May. RAX, the PKR activator, sensitizes cells to inflammatory cytokines, serum withdrawal, chemotherapy, and viral infection. Blood 2006; 108: 821-829.
3 Hsu LC, Park JM, Zhang K, Luo JL, Maeda S, Kaufman RJ et al. The protein kinase PKR is required for macrophage apoptosis after activation of Toll-like receptor 4. Nature 2004; 428: 341-345.

4 Li YJ, Zeng JM, Huang SF, Wang XZ, Zhao SQ, Bai WJ et al. Selective leukemia cell death by activation of the double-stranded RNA-dependent protein kinase PKR. Int J Mol Med 28: 215-222.

5 Murad JM, Tone LG, de Souza LR, De Lucca FL. A point mutation in the RNAbinding domain I results in decrease of PKR activation in acute lymphoblastic leukemia. Blood Cells Mol Dis 2005; 34: 1-5.

6 Liu X, Bennett RL, Cheng X, Byrne M, Reinhard MK, May Jr WS. PKR regulates proliferation, differentiation, and survival of murine hematopoietic stem/progenitor cells. Blood 2013; 121: 3364-3374.

7 Bennett RL, Pan Y, Christian J, Hui T, May Jr WS. The RAX/PACT-PKR stress response pathway promotes p53 sumoylation and activation, leading to $G(1)$ arrest. Cell Cycle 2012; 11: 407-417.

8 Ruvolo VR, Kurinna SM, Karanjeet KB, Schuster TF, Martelli AM, McCubrey JA et al. PKR regulates B56(alpha)-mediated BCL2 phosphatase activity in acute lymphoblastic leukemia-derived REH cells. J Biol Chem 2008; 283: 35474-35485.

9 Deb A, Haque SJ, Mogensen T, Silverman RH, Williams BR. RNA-dependent protein kinase PKR is required for activation of NF-kappa B by IFN-gamma in a STAT1independent pathway. J Immunol 2001; 166: 6170-6180.

10 Goh KC, deVeer MJ, Williams BR. The protein kinase PKR is required for p38 MAPK activation and the innate immune response to bacterial endotoxin. EMBO $J$ 2000; 19: 4292-4297.

11 Abraham N, Jaramillo ML, Duncan PI, Methot N, Icely PL, Stojdl DF et al. The murine PKR tumor suppressor gene is rearranged in a lymphocytic leukemia. Exp Cell Res 1998; 244: 394-404.

12 Beretta L, Gabbay M, Berger R, Hanash SM, Sonenberg N. Expression of the protein kinase PKR in modulated by IRF-1 and is reduced in $5 q$ - associated leukemias. Oncogene 1996; 12: 1593-1596.

13 Blalock WL, Bavelloni A, Piazzi M, Faenza I, Cocco L. A role for PKR in hematologic malignancies. J Cell Physiol 223: 572-591.

14 Hii Sl, Hardy L, Crough T, Payne EJ, Grimmett K, Gill D et al. Loss of PKR activity in chronic lymphocytic leukemia. Int J Cancer 2004; 109: 329-335.

15 Yeung MC, Liu J, Lau AS. An essential role for the interferon-inducible, doublestranded RNA-activated protein kinase PKR in the tumor necrosis factor-induced apoptosis in U937 cells. Proc Natl Acad Sci USA 1996; 93: 12451-12455.

16 Pataer A, Raso MG, Correa AM, Behrens C, Tsuta K, Solis L et al. Prognostic significance of RNA-dependent protein kinase on non-small cell lung cancer patients. Clin Cancer Res 2010; 16: 5522-5528.

17 Savinova O, Joshi B, Jagus R. Abnormal levels and minimal activity of the dsRNAactivated protein kinase, PKR, in breast carcinoma cells. Int J Biochem Cell Biol 1999; 31: 175-189.

18 Bennett RL, Carruthers AL, Hui T, Kerney KR, Liu X, May Jr WS. Increased expression of the dsRNA-activated protein kinase PKR in breast cancer promotes sensitivity to doxorubicin. PLoS One 2012; 7: e46040.

19 Kim SH, Forman AP, Mathews MB, Gunnery S. Human breast cancer cells contain elevated levels and activity of the protein kinase, PKR. Oncogene 2000; 19: 3086-3094.

20 Barber GN, Wambach M, Thompson S, Jagus R, Katze MG. Mutants of the RNAdependent protein kinase (PKR) lacking double-stranded RNA binding domain I can act as transdominant inhibitors and induce malignant transformation. Mol Cell Biol 1995; 15: 3138-3146.

21 Donze O, Jagus R, Koromilas AE, Hershey JW, Sonenberg N. Abrogation of translation initiation factor elF-2 phosphorylation causes malignant transformation of NIH 3T3 cells. EMBO J 1995; 14: 3828-3834.

22 Meurs EF, Galabru J, Barber GN, Katze MG, Hovanessian AG. Tumor suppressor function of the interferon-induced double-stranded RNA-activated protein kinase. Proc Natl Acad Sci USA 1993; 90: 232-236.

23 Wickremasinghe RG, Hoffbrand AV. Biochemical and genetic control of apoptosis: relevance to normal hematopoiesis and hematological malignancies. Blood 1999; 93: 3587-3600.

$24 \mathrm{Xu} \mathrm{Z}$, Williams BR. The B56alpha regulatory subunit of protein phosphatase $2 \mathrm{~A}$ is a target for regulation by double-stranded RNA-dependent protein kinase PKR. Mol Cell Biol 2000; 20: 5285-5299.

25 Ruvolo PP, Deng X, May WS. Phosphorylation of $\mathrm{Bcl} 2$ and regulation of apoptosis. Leukemia 2001; 15: 515-522.

26 Deng X, Gao F, May WS. Protein phosphatase 2A inactivates Bcl2's antiapoptotic function by dephosphorylation and up-regulation of $\mathrm{Bcl} 2-\mathrm{p} 53$ binding. Blood 2009; 113: 422-428.

27 Wang YY, Deng X, Xu L, Gao F, Flagg T, May WS. Bcl2 enhances induced hematopoietic differentiation of murine embryonic stem cells. Exp Hematol 2008; 36: 128-139.

28 Deng X, Gao F, Flagg T, May Jr. WS. Mono- and multisite phosphorylation enhances Bcl2's antiapoptotic function and inhibition of cell cycle entry functions. Proc Natl Acad Sci USA 2004; 101: 153-158. 
29 May WS, Tyler PG, Ito T, Armstrong DK, Qatsha KA, Davidson NE. Interleukin-3 and bryostatin-1 mediate hyperphosphorylation of BCL2 alpha in association with suppression of apoptosis. J Biol Chem 1994; 269: 26865-26870.

30 Ruvolo PP, Clark W, Mumby M, Gao F, May WS. A functional role for the B56 alphasubunit of protein phosphatase $2 \mathrm{~A}$ in ceramide-mediated regulation of $\mathrm{Bcl} 2$ phosphorylation status and function. J Biol Chem 2002; 277: 22847-22852.

31 Neviani P, Santhanam R, Oaks JJ, Eiring AM, Notari M, Blaser BW et al. FTY720, a new alternative for treating blast crisis chronic myelogenous leukemia and Philadelphia chromosome-positive acute lymphocytic leukemia. J Clin Invest 2007; 117: 2408-2421.

32 Matsuoka Y, Nagahara Y, Ikekita M, Shinomiya T. A novel immunosuppressive agent FTY720 induced Akt dephosphorylation in leukemia cells. Br J Pharmacol 2003; 138: 1303-1312.

33 Perrotti D, Neviani P. Protein phosphatase 2A (PP2A), a drugable tumor suppressor in Ph1(+) leukemias. Cancer Metastasis Rev 2008; 27: 159-168.

34 Wallington-Beddoe CT, Don AS, Hewson J, Qiao Q, Papa RA, Lock RB et al. Disparate in vivo efficacy of FTY720 in xenograft models of Philadelphia positive and negative B-lineage acute lymphoblastic leukemia. PLoS One 2012; 7: e36429.

35 Yang Y, Huang Q, Lu Y, Li X, Huang S. Reactivating PP2A by FTY720 as a nove therapy for AML with C-KIT tyrosine kinase domain mutation. J Cell Biochem 2012 113: $1314-1322$
36 Bialojan C, Takai A. Inhibitory effect of a marine-sponge toxin, okadaic acid on protein phosphatases. Specificity and kinetics. Biochem J 1988; 256: 283-290.

37 Mai H, May WS, Gao F, Jin Z, Deng X. A functional role for nicotine in Bcl2 phosphorylation and suppression of apoptosis. J Biol Chem 2003; 278: 1886-1891.

38 Ettenger R, Schmouder R, Kovarik JM, Bastien MC, PF Hoyer. Pharmacokinetics, pharmacodynamics, safety, and tolerability of single-dose fingolimod (FTY720) in adolescents with stable renal transplants. Pediatr Transplant 2011; 15: 406-413.

39 Fazekas F. Fingolimod in the treatment algorithm of relapsing remitting multiple sclerosis: a statement of the Central and East European (CEE) MS Expert Group. Wien Med Wochenschr 2012; 162: 354-366.

40 Seshacharyulu P, Pandey P, Datta K, Batra SK. Phosphatase: PP2A structural importance, regulation and its aberrant expression in cancer. Cancer Lett 2013; 335: 9-18.

(c) (i) $\Theta$ This work is licensed under a Creative Commons AttributionNonCommercial-NoDerivs 3.0 Unported License. To view a copy of this license, visit http://creativecommons.org/licenses/by-nc-nd/3.0/ 